\title{
Radiation dose escalation by simultaneous modulated accelerated radiotherapy combined with chemotherapy for esophageal cancer: a phase II study
}

\author{
Jianzhou Chen ${ }^{1,2, *}$, Hong Guo ${ }^{1, *}$, Tiantian Zhai ${ }^{1}$, Daniel Chang ${ }^{3}$, Zhijian Chen ${ }^{4}$, \\ Ruihong Huang ${ }^{1}$, Wuzhe Zhang ${ }^{1}$, Kun Lin ${ }^{5}$, Longjia Guo ${ }^{1}$, Mingzhen Zhou ${ }^{1}$, \\ Dongsheng Li ${ }^{1}$, Derui Li ${ }^{1}$, Chuangzhen Chen ${ }^{1}$ \\ ${ }^{1}$ Department of Radiation Oncology, Cancer Hospital of Shantou University Medical College, Shantou, Guangdong, China \\ ${ }^{2}$ CRUK/MRC Oxford Institute for Radiation Oncology, University of Oxford, Oxford, United Kingdom \\ ${ }^{3}$ Department of Radiation Oncology, Stanford University, Stanford, CA, USA \\ ${ }^{4}$ Department of Oncology, The University of Hongkong - Shenzhen Hospital, Shenzhen, Guangdong, China \\ ${ }^{5}$ Department of Public Health and Preventive Medicine, Shantou University Medical College, Shantou, Guangdong, China \\ *These authors have contributed equally to this work \\ Correspondence to: Chuangzhen Chen, e-mail: stccz@139.com \\ Dervi Li, e-mail: dervi_li@aliyun.com \\ Keywords: esophageal cancer, radiation therapy, dose escalation, simultaneous modulated accelerated radiotherapy, \\ simultaneous integrated boost \\ Received: November 24, $2015 \quad$ Accepted: February 23, $2016 \quad$ Published: March 14, 2016
}

\section{ABSTRACT}

The outcomes for patients with esophageal cancer (EC) underwent standarddose radical radiotherapy were still disappointing. This phase II study investigated the feasibility, safety and efficacy of radiation dose escalation using simultaneous modulated accelerated radiotherapy (SMART) combined with chemotherapy in 60 EC patients. Radiotherapy consisted of $66 \mathrm{~Gy}$ at $2.2 \mathrm{~Gy} /$ fraction to the gross tumor and 54Gy at $1.8 \mathrm{~Gy} /$ fraction to subclinical diseases simultaneously. Chemotherapy including cisplatin and 5 fluorouracil were administered to all patients during and after radiotherapy. The data showed that the majority of patients $(98.3 \%)$ completed the whole course of radiotherapy and concurrent chemotherapy. The most common $\geq$ grade 3 acute toxicities were neutropenia (16.7\%), followed by esophagitis $(6.7 \%)$ and thrombopenia (5.0\%). With a median follow-up of 24 months (5-38) for all patients and 30 months (18-38) for those still alive, 11 patients $(18.3 \%)$ developed $\geq$ Grade 3 late toxicities and $2(3.3 \%)$ of them died subsequently due to esophageal hemorrhage. The 1- and 2-year local-regional control, distant metastasis-free survival, disease-free survival and overall survival rates were $87.6 \%$ and $78.6 \%, 86.0 \%$ and $80.5 \%, 75.6 \%$ and $64.4 \%, 86.7 \%$ and $72.7 \%$, respectively. SMART combined with concurrent chemotherapy is feasible in EC patients with tolerable acute toxicities. They showed a trend of significant improvements in local-regional control and overall survival. Further follow-up is needed to evaluate the late toxicities.

\section{INTRODUCTION}

Esophageal cancer (EC) is one of the most deadliest malignancies. The current standard of nonsurgical treatment for this disease is 50 Gray (Gy) of radiotherapy (RT) in conventional fractionation with concurrent chemotherapy. This regimen has been established since the 1990s [1, 2], though $50 \%$ of patients still had local failure after treatment.
The Intergroup 0123 trial (INT0123) was undertaken to investigate the potential benefits of higher RT dose: 64.8Gy vs. 50.4Gy [3]. However, long-term follow-up showed that it did not improve the 2-year overall survival (OS: 31\% vs. 40\%) and local-regional control rates (LRC: $46 \%$ vs. $48 \%$ ). Therefore, to date, the standard treatment for EC is still 50 Gy of conventionally fractionated RT with concurrent chemotherapy. 
Intensity modulated radiotherapy (IMRT) is a modern high-precision RT technique which can reduce the dose to organs at risk (OARs) while maintaining the tumor coverage. A retrospective study demonstrated that EC patients treated with IMRT had less non cancer-related deaths, better LRC and OS, compared with patients treated with three-dimensional conformal radiotherapy (3DCRT) [4]. Meanwhile, there are also some studies suggesting that positive correlation between RT dose and LRC may exist in EC patients [5, 6]. Thus, it is logic to assume that patients with EC may have better LRC if treated with RT dose escalation using modern delivery technique.

Simultaneous modulated accelerated radiotherapy (SMART), also known as simultaneous integrated boost (SIB), is a novel dose escalation technique with IMRT, by which different dose fractionation could be delivered to OARs and tumors simultaneously. The smaller fraction size $(<2 \mathrm{~Gy})$ in OARs helps further reduce the risk and severity of toxicities, while the larger fraction size (>2Gy) in tumors results in shorter treatment time and higher biologically equivalent dose. It is well acknowledged that tumor clonogen proliferation during conventional RT is a significant factor responsible for local failure of squamous cell carcinoma (SCC) of the upper respiratory and digestive tracts $[7,8]$. A shorter overall treatment time of RT has been shown to be more beneficial to the treatment of EC $[9,10]$. Additionally, one study found that the estimated alpha/beta of EC was 4.9 Gy which was lower than commonly expected, suggesting that hypofractionated RT may be more biologically effective for EC [6].

Taken together, we hypothesized that it was feasible to perform RT dose escalation with modest hypofractionation in EC patients by taking advantage of the better sparing of OARs using SMART technique. This was first supported by a dosimetric study reported previously [11]. We then began a single-arm phase II trial in 2012 to investigate the application of SMART combined with concurrent chemotherapy in EC (Clinicaltrial. gov number, NCT01670409, and Chinese Clinical Research Registry number, ChiCTR-ONC-12002356). The feasibility, safety and efficacy of this regimen in 60 enrolled patients were summarized here.

\section{RESULTS}

\section{Patient characteristics}

Between August 2012 and April 2014, a total of 60 patients were enrolled in this phase II trial. Patient characteristics were listed in Table 1 . The majority of patients $(78.3 \%)$ had locally advanced diseases $\left(\mathrm{T}_{3 / 4}\right)$. The percentage of patients with positive lymph nodes (LNs) was $61.7 \%$. Among the 60 patients, $11(18.3 \%)$ of them were in stage IV due to supraclavicular LN metastasis.

\section{Quality review of treatment plans}

Dose-volume parameters for planning target volumes (PTVs) were summarized in Table 2 according to International Commission on Radiation Units \& Measurements 83 report [12]. The median $\mathrm{V}_{20}$ for the lungs and $\mathrm{D}_{\text {mean }}$ for the heart were $15.3 \%(3.8 \sim 32.2 \%)$ and 11.0 Gy (0-38.9Gy) respectively. Planning objectives were well met in all patients.

\section{Treatment completion}

All patients finished the entire planned course of RT, except one $(1.7 \%)$ who received $97 \%$ of the prescribed dose due to grade 4 thrombocytopenia. The numbers of chemotherapy cycle that the patients completed were as follows: 4 cycles, 47 cases (78.3\%); 3 cycles, 6 cases (10.0 $\%) ; 2$ cycles, 6 cases $(10.0 \%)$ and 1 cycle, 1 case $(1.7 \%)$. Fifty-nine patients (98.3\%) finished at least the concurrent chemotherapy. The main reasons that patients did not receive the remaining chemotherapy included severe haematological toxicities, esophagitis and patient's refusal. There were no dose modifications of chemotherapy. One patient $(1.7 \%)$ underwent exploratory thoracotomy plus gastrostomy after completion of RT and 2 cycles of concurrent chemotherapy based on his own decision.

\section{Acute toxicities}

The majority of patients only experienced mild acute toxicities (Table 3 ). The most common $\geq$ grade 3 acute toxicities were neutropenia (16.7\%), followed by esophagitis $(6.7 \%)$ and thrombopenia $(5.0 \%)$. No treatment-related death was documented in the first 3 months since the beginning of treatment.

\section{Late toxicities}

The last follow-up was October 10, 2015. As of this writing, 17 of $60(28.3 \%)$ patients have died. The median follow-up was 24 months (5-38 months) for all patients and 30 months (18-38 months) for those still alive. The follow-up rate was $100 \%$. The percentages of late toxicities were summarized in Table 4 . Eleven patients (18.3\%) developed $\geq$ Grade 3 late toxicities: 9 patients $(15.0 \%)$ at the esophagus and the other $2(3.3 \%)$ at lungs. Esophageal ulcers/fistulas were all found within the radiation dose escalation region. Two patients (3.3\%) died subsequently due to esophageal hemorrhage. No severe late toxicities of skin, heart, spinal cord, hematologic system and liver had been reported.

\section{Failure patterns and survivals}

There were no persistent diseases in all enrolled patients evaluated by contrast-enhanced computer tomography (CT) and barium swallow test. Within the 
Table 1: Clinical characteristics of $60 \mathrm{EC}$ patients who received SMART combined with chemotherapy

\begin{tabular}{lccc}
\hline Characteristics & & No. & \% \\
\hline Age & Male & Median 62 years (45-73) & \\
Gender & Female & 50 & 83.3 \\
& 1 & 10 & 16.7 \\
T stage* & 2 & 1 & 1.7 \\
& 3 & 12 & 20.0 \\
& 4 & 32 & 53.3 \\
N stage* & 0 & 15 & 25 \\
& 1 & 23 & 38.3 \\
M stage* & 0 & 37 & 61.7 \\
& $1_{\mathrm{a}}$ & 49 & 81.7 \\
Clinical stage* & $1_{\mathrm{b}}$ & 3 & 5.0 \\
& $\mathrm{II}_{\mathrm{A}}$ & 8 & 13.3 \\
& $\mathrm{II}_{\mathrm{B}}$ & 18 & 30.0 \\
& $\mathrm{III}$ & 4 & 6.7 \\
& $\mathrm{IV}$ & 27 & 45.0 \\
Lesion site & $\mathrm{IV}_{\mathrm{b}}$ & 3 & 5.0 \\
& Cervical & 8 & 13.3 \\
& Upper thoracic & 4 & 6.7 \\
& Middle thoracic & 25 & 41.7 \\
& & 31 & 51.7 \\
\hline
\end{tabular}

Abbreviations: EC: esophageal cancer. SMART: simultaneous modulated accelerated radiotherapy.

* According to American Joint Committee on Cancer (AJCC) staging system $6^{\text {th }}$.

Table 2: Dose-volume parameters of PTVs for the 60 EC patients who received SMART combined with chemotherapy

\begin{tabular}{lcc}
\hline Parameters Median (range) & PTV $_{66}$ & PTV $_{54}$ \\
\hline Volume $\left(\mathrm{cm}^{3}\right)$ & $74.3(13.5-212.0)$ & $199.5(97.5-750.7)$ \\
$\mathrm{D}_{2}(\mathrm{~Gy})$ & $69.5(67.5-71.5)$ & $67.7(59.6-70.6)$ \\
$\mathrm{D}_{98}(\mathrm{~Gy})$ & $65.2(62.9-66.1)$ & $53.1(50.4-55.7)$ \\
$\mathrm{D}_{50}(\mathrm{~Gy})$ & $68.1(66.8-69.9)$ & $59.2(56.1-62.9)$ \\
$\mathrm{HI}$ & $0.07(0.03-0.11)$ & $0.24(0.11-0.30)$ \\
$\mathrm{CI}$ & $0.81(0.63-1.2)$ & $0.79(0.59-0.88)$ \\
\hline
\end{tabular}

Abbreviation: PTV: planning target volume. EC: esophageal cancer. SMART: simultaneous modulated accelerated radiotherapy. Dx was defined as the minimum dose to a specified target volume. $H I$ : Homogeneity Index. $H I=\mathrm{D}_{5} / \mathrm{D}_{95}$. $\mathrm{D}_{5}$ and $\mathrm{D}_{95}$ were the minimum doses received by the hot $5 \%$ and cold $95 \%$ of PTV, respectively.

$C I$ : Conformity Index. $C I=\frac{V_{T, r e f}}{V_{T}} \times \frac{V_{T, r e f}}{V_{r e f}} . V_{T}$ : The target volume. $V_{T, r e f}$ : The target volume covered by reference isodose. $V_{r e f}:$ The total volume covered by reference isodose. 
Table 3: Acute toxicities of the 60 EC patients who received SMART combined with chemotherapy

\begin{tabular}{lccc}
\hline Toxicities No. (\%) & Grade3 & Grade4 & Grade5 \\
\hline Neutropenia & $5(8.3 \%)$ & $5(8.3 \%)$ & 0 \\
Esophagitis & $4(6.7 \%)$ & 0 & 0 \\
Thrombopenia & $2(3.3 \%)$ & $1(1.7 \%)$ & 0 \\
Nausea/Vomiting & $2(3.3 \%)$ & 0 & 0 \\
Anemia & $2(3.3 \%)$ & 0 & 0 \\
Others & 0 & 0 & 0 \\
\hline
\end{tabular}

Abbreviations: EC: esophageal cancer. SMART: simultaneous modulated accelerated radiotherapy.

* Graded by Common Terminology Criteria for Adverse Events Version 4.0.

Table 4: Late toxicities of the 60 EC patients who received SMART combined with chemotherapy

\begin{tabular}{lccc}
\hline Toxicities No. $(\%)$ & Grade3 & Grade4 & Grade5 \\
\hline Esophageal ulcer/fistula & $5(8.3 \%)$ & 0 & $2(3.3 \%)$ \\
Esophageal stricture & $3(5.0 \%)$ & 0 & 0 \\
Lung & $2(3.3 \%)$ & 0 & 0 \\
Others & 0 & 0 & 0 \\
\hline
\end{tabular}

Abbreviations: EC: esophageal cancer. SMART: simultaneous modulated accelerated radiotherapy.

follow-up time, tumor failure occurred in 20 patients $(33.3 \%)$. The first site of failure was listed in Table 5 . The most common pattern was distant metastasis $(18.3 \%)$, followed by regional recurrence (11.7\%) and local recurrence $(8.3 \%)$. The LRC and survival curves were shown in Figure 1. The 1-year and 2-year LRC, distant metastasis-free survival (DMFS), disease-free survival (DFS), and OS rates were $87.6 \%$ and $78.6 \%, 86.0 \%$ and $80.5 \%, 75.6 \%$ and $64.4 \%, 86.7 \%$ and $72.7 \%$, respectively.

\section{DISCUSSION}

Emerging dosimetric data suggests that RT dose escalation using modern delivery techniques may have a potential to improve the LRC of EC, however, the feasibility and efficacy of such approach have been unclear $[11,13,14]$. To our knowledge, this is the first report of phase II study of radiation dose escalation using simultaneous boost approach (SMART) for the treatment of EC. We found that it is feasible to apply SMART with concurrent chemotherapy in EC patients. Moreover, patients enrolled in this study showed a trend of significant improvements in LRC and OS.

The majority of patients in this study experienced mild acute toxicities and consistently, showed excellent compliance of treatment. Among the 60 patients, $98.3 \%$ and $78.3 \%$ of them finished the whole course of RT and chemotherapy, respectively, whereas only $67 \%$ and $66 \%$ of patients in the higher-dose arm of INT0123 trial completed RT and chemotherapy according to the protocol, respectively [3]. The treatment completion of our study is also comparable to patients underwent standarddose RT using 3DCRT combined with different regimens of chemotherapy [15]. A couple of factors may contribute to the excellent treatment completion, including better sparing of OARs using SMART technique, lesser amount of 5Fluorouracil (5-Fu) administrated and smaller RT field.

In regard to late toxicities, we found that $18.3 \%$ of patients had $\geq$ Grade 3 late toxicities within the follow-up period. The rates of grade 4 and grade 5 toxicities were $0 \%$ and $3.3 \%$, respectively, as compared to $8 \%$ and $2 \%$ in the combined modality arm of RTOG $85-01$, and $24 \%$ and $10 \%$ in RTOG 92-07 $[1,16]$. Thus, in comparison to histological data, our concurrent results do not suggest that SMART would significantly increase the risk of life-threatening toxicities and treatment-related death either. However, it should be noted that the follow-up in this study is relatively shorter than that in RTOG 85-01. Therefore, to more comprehensively evaluate the late toxicities, further follow-up is needed.

In line with the $3.3 \%$ of treatment-related death, patients enrolled in this study showed a trend of significant improvements in local tumor control and OS compared with previous studies, which is very striking. Only $8.4 \%$ of patient experienced local recurrences with a median follow-up of 30 months. The 2-year LRC and OS rates were $78.6 \%$ and $72.7 \%$, respectively, as compared to $48 \%$ 
Table 5: Failure patterns of 20 out of the 60 patients who received SMART combined with chemotherapy

\begin{tabular}{lcc}
\hline First sites of failure & No & \% \\
\hline Distant metastasis & 10 & 16.7 \\
Regional recurrence & 4 & 6.7 \\
Local recurrence & 4 & 6.7 \\
Local and regional recurrence & 1 & 1.7 \\
Regional and distant & 1 & 1.7 \\
\hline
\end{tabular}

Abbreviations: EC: esophageal cancer. SMART: simultaneous modulated accelerated radiotherapy.

A

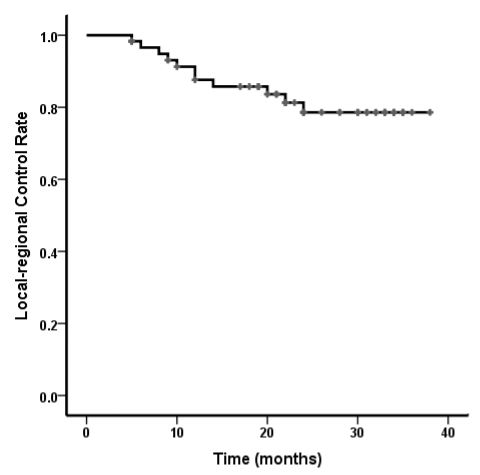

$\mathrm{C}$

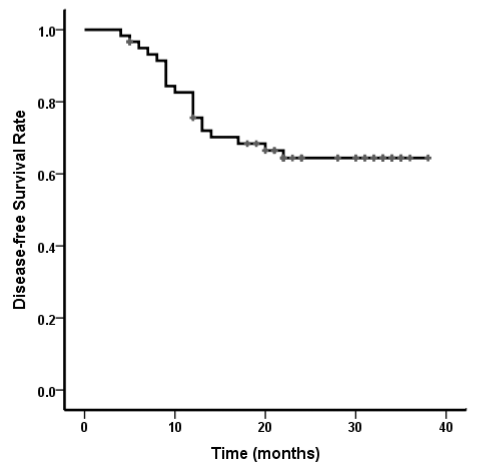

B

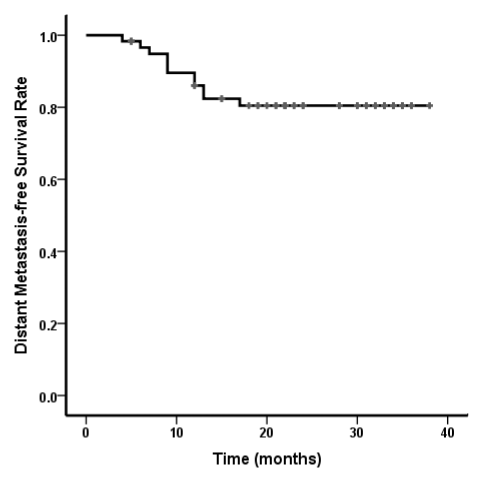

D

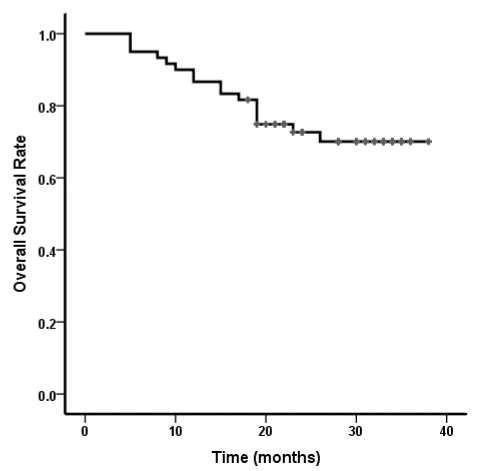

Figure 1: Local-regional control and survival curves of the $60 \mathrm{EC}$ patients who received SMART combined with chemotherapy. A. Local-regional control rate; B. Distant-metastasis free survival rate; C. Disease-free survival rate; D. Overall survival rate. Abbreviations: EC: esophageal cancer. SMART: simultaneous modulated accelerated radiotherapy.

and $40 \%$ in the standard-dose arm of INT0123, despite more patients enrolled in this trial were in advanced stages: $\mathrm{T}_{3 / 4}$ stage $(78.3 \%$ vs. $43 \%), \mathrm{N}_{1}(61.7 \%$ vs. $17 \%)$ and $\mathrm{M}_{1}(18.3 \%$ vs. $0 \%)$ [3]. Apart from cancer stage, other factors, including target definition and radiation delivery technique, may also affect the outcomes of patients. The GTV in our study was determined by endoscopic ultrasonography (EUS), in addition to CT and esophageal barium swallow test that were used in INT0123, which may potentially reduce the risk of missing the target, resulting in better tumor control $[17,18]$. However, the majority of local recurrences in INT0123 were actually within the GTV. Thus, it less likely that the improvement of tumor control in this study was derived from better target definition. Regarding the radiation delivery technique, IMRT and IGRT were applied in this study as compared to conventional RT technique in INT0123. Although advanced RT techniques could provide more accurate and precise treatment delivery, it is still unclear whether these advantages would lead to improvements 
in local tumor control and overall survival [4, 19-21]. Nevertheless, phase III trials comprising stand-dose arm using modern RT technique are warranted.

The dose escalation regimen applied in this trial was $66 \mathrm{~Gy}$ in $2.2 \mathrm{~Gy} / \mathrm{F}$. Whether further dose escalation is tolerable and beneficial to the patients is unclear. In a recently published phase I dose escalation study, Wen Yu, et al, suggested that it was feasible to deliver up to 62.5 Gy $(2.5 \mathrm{~Gy} / \mathrm{F})$ to GTV and $70 \mathrm{~Gy}(2.8 \mathrm{~Gy} / \mathrm{F})$ to the high risk region of GTV based on PET/CT, respectively, in EC patients [22]. However, the safety evaluation of dose escalation in this study relied mainly on acute toxicities without the information of late toxicities. Besides, in each dose escalation level, only 5 patients were recruited, whereas life-threatening esophagitis generally occurs in relatively smaller rate and requires larger sample size to evaluate. More importantly, of the 60 patients enrolled in our study, only 5 of them $(8.4 \%)$ had local recurrence, while there were already 2 cases $(3.3 \%)$ of treatment-related death due to esophageal hemorrhage. Thus, it is more likely that further dose escalation may lead to increased risk of treatment-related death, rather than better local tumor control.

One pitfall of this study is the lack of standard control. Comparison of SMART and standard-dose IMRT should be performed in future phase III trial. Additionally, all patients in this study were recruited in one single center. Whether this dose escalation regimen could be applied in different centers remains to be determined.

In summary, SMART combined with concurrent chemotherapy is feasible in EC patients with tolerable acute toxicities. They showed a trend of significant improvements in LRC and OS. Further follow-up is needed to evaluate the late toxicities. Phase III randomized trial is warranted to compare this regimen with standarddose RT.

\section{MATERIALS AND METHODS}

\section{Ethic statement}

This study was in accordance with the Helsinki Declaration (2000) and was approved by the Clinical Research Ethics Review Committee of Cancer Hospital of Shantou University Medical College. Study-specific written informed consents were obtained from all patients prior to enrolment.

\section{Patient selection}

Inclusion criteria were as follows: (a) pathologically proven primary esophageal SCC; (b) disease located in cervical, upper or middle thoracic esophagus; (c) no distant metastases (except supraclavicular lymph node); (d) Zubrod performance status: 0 2; (e) adequate liver, renal and bone marrow function; (f) women of childbearing potential and male participants must practice adequate contraception.
Exclusion criteria included: (a) evidence of tracheoesophageal or mediastinal- esophageal fistula; (b) prior invasive malignancy (except non-melanomatous skin cancer) unless disease-free for a minimum of 2 years; (c) prior RT that would result in overlap of the planned RT fields; (d) severe and active comorbidities; (e) pregnant or nursing women.

\section{Pretreatment evaluation}

The following evaluations were performed: medical history and physical examination, EUS of esophagus with biopsy, esophagography with barium swallow or iopromide (water-soluble nonionic contrast medium) when esophageal fistula may be present, plain and contrast-enhanced CT scan from the neck to the upper abdomen, abdominal ultrasound, electrocardiogram, hematologic and biochemical profiles. Bronchial endoscopies, bone scans and Positron emission tomography-CT (PET/CT) scans were performed as clinically indicated. All patients were staged using the American Joint Cancer Committee (AJCC) staging system $6^{\text {th }}[23]$.

\section{Radiotherapy}

\section{Immobilization and $\mathrm{CT}$ simulation}

Patients were immobilized in supine position with the head and shoulders encompassed in a thermoplastic shell. Contrast-enhanced CT scan (3mm slice thickness) from the neck to the upper abdomen was obtained using a 16-slice CT scanner (The Philips Brilliance CT Big Bore Oncology Configuration, Cleveland, OH). CT images were then delivered to the Eclipse 10.0 treatment planning system (Varian Medical Systems, Palo Alto, CA) for target volume, OARs contouring and subsequent treatment planning.

\section{Target volumes and prescribed doses}

We have reported our treatment planning approach previously [11]. The gross tumor volume (GTV) includes the primary tumor $\left(\mathrm{GTV}_{\mathrm{p}}\right)$ of esophagus and positive regional lymph nodes $\left(\mathrm{GTV}_{\mathrm{LN}}\right)$. The contour of GTV was determined by CT images, endoscopic reports or barium/ iopromide swallow fluoroscopy whichever larger. $\mathrm{GTV}_{\mathrm{LN}}$ includes mediastinal or supraclavicular LNs if the shortest axis $\geq 1 \mathrm{~cm}$. Clinical target volume (CTV) was delineated with $2-\mathrm{cm}$ longitudinal and $0.5-$ to $1.0-\mathrm{cm}$ radial margins with respect to the $\mathrm{GTV}_{\mathrm{P}}$ and a $0.5-\mathrm{cm}$ uniform margin for $\mathrm{GTV}_{\mathrm{LN}}$. Paraesophageal or tracheoesophageal groove LNs that did not meet the criteria of positive LN but their shortest axis $\geq 0.5 \mathrm{~cm}$ were also encompassed in CTV. No other regional LNs were included in CTV for prophylactic irradiation. Two $\mathrm{PTV}_{\mathrm{S}}$ were derived from the GTV and CTV, respectively: $\mathrm{PTV}_{66}=\mathrm{GTV}+0.5 \mathrm{~cm}$ and $\mathrm{PTV}_{54}=\mathrm{CTV}+0.5 \mathrm{~cm}$. The prescribed dose was 
$66 \mathrm{~Gy} / 30 \mathrm{~F}$ to $\mathrm{PTV}_{66}(2.2 \mathrm{~Gy} / \mathrm{F})$ and $54 \mathrm{~Gy} / 30 \mathrm{~F}$ to $\mathrm{PTV}_{54}$ $(1.8 \mathrm{~Gy} / \mathrm{F})$ in a single plan. OAR contours were created for the spinal cord, lungs, and heart.

\section{Planning objectives}

The planning objectives for PTV were $100 \%$ of the PTV volume receiving $95 \%$ of the prescribed dose. The dose constraints for OARs were as follows: spinal cord, $\mathrm{D}_{\text {max }}$ (maximum dose) $<45 \mathrm{~Gy}$; heart, $\mathrm{V}_{40}\left(\mathrm{~V}_{\mathrm{x}}=\right.$ percentage of the target volume receiving $\geq \mathrm{x}$ Gy) $<100 \%, \mathrm{~V}_{45}<67 \%$ and $\mathrm{V}_{50}<33 \%$; lungs, $\mathrm{V}_{20}<30 \%, \mathrm{~V}_{10}<50 \%$ and $\mathrm{V}_{5}<$ $60 \%$.

\section{Planning techniques}

The SMART plans were generated using a sliding window dynamic delivery with 5 coplanar beams (angles: $\left.210^{\circ} / 300^{\circ} / 0^{\circ} / 60^{\circ} / 150^{\circ}\right)$. All plans were designed to be delivered using 6-MV photon beams from a linear accelerator (TrueBeam, Varian Medical Systems, Palo Alto, CA). Plans were optimized, selecting a maximum dose rate of $600 \mathrm{MU} / \mathrm{min}$. Dose calculation was performed using Anisotropic Analytical Algorithm 8.6.02 with lung heterogeneity correction.

\section{Image-guided radiotherapy}

Cone-beam CT (CBCT) scans were obtained in all patients prior to treatment delivery to assess potential setup errors at least once per week. Whenever the setup error is more than $5 \mathrm{~mm}$ in any dimension, surface markers on the thermoplastic shell that were used to align with radiation isocenter would be adjusted accordingly to correct the setup error. Adjustments would need to be validated in the next fraction of treatment. Re-simulation and replanning would be considered if the setup error could not be corrected or the relationship between the tumor and adjacent critical structures changed significantly.

\section{Chemotherapy}

All patients were treated with 2 cycles of concurrent chemotherapy on days 1 and 29, and 2 cycles of adjuvant chemotherapy on days 50 and 71 . The chemotherapy regimen was as follows: cisplatin, $75 \mathrm{mg} / \mathrm{m}^{2}$, intravenous on day $1,5-\mathrm{Fu} 0.5 \mathrm{~g} / \mathrm{m}^{2}$, intravenous drip infusion on day 1 to 4 .

\section{Dose modification}

Dose modification of RT and chemotherapy was based on patient toxicities using common terminology criteria for adverse events (CTCAE 4.0) [24].

RT was delivered only when patients fulfilled the following conditions: (a) neutrophils $\geq 1.0 \times 10^{9} / \mathrm{L}$; (b) blood platelet $\geq 50 \times 10^{9} / \mathrm{L}$; (c) No grade 4 acute toxicities For patients with grade 4 non-haematological toxicities, the remaining RT would be cancelled. RT would be suspended in patients with grade 3 non-haematological toxicities until they recovered. Patients could still receive treatment with $\leq$ grade 2 non-haematological toxicities unless further treatment would augment the severity of toxicities.

Chemotherapy was prescribed only when patients fulfilled the following conditions: (a) neutrophils $\geq$ $2.0 \times 10^{9} / \mathrm{L}$ or $\geq 1.5 \times 10^{9} / \mathrm{L}$ for appropriate patients without fever; (b) blood platelet $\geq 100 \times 10^{9} / \mathrm{L}$; (c) no grade $\geq 3$ chemotherapy-related toxicities. For patients with grade 3 toxicities, chemotherapy should be postponed until the toxicities relieved. When patients experienced the first time of grade 4 bone marrow suppression, the dose of next cycle of chemotherapy would be reduced to $75 \%$ of the prescribed dose. The chemotherapy would be cancelled if patients had experienced twice of grade 4 bone marrow suppression. Carboplatin would be used to replace cisplatin if patients had significant nephro-, oto-, or neuro-toxicity.

\section{Assessment of acute toxicities}

All patients were hospitalized during the treatment and assessed every week for acute toxicities using CTCAE 4.0. Routine evaluations included physical exam, hematologic and biochemical profiles, and esophagography (every 2 weeks during RT and once in each cycle of adjuvant chemotherapy).

\section{Follow-up}

Patients were assessed at 3 months since the beginning of treatment for short-term efficacy, and then every 3 months for 2 years and every 6 months for 3 years. History, physical examination, hematologic and biochemical profiles, late toxicities assessment, chest X-ray plus esophagography or contrast-enhanced CT scan, and abdominal ultrasound were to be done at each visit. EUS of esophagus with biopsy or PET/CT was performed if clinically indicated. Local recurrence should be confirmed with pathological proof or at least two imaging examinations when biopsy was not applicable.

\section{Endpoints and sample size}

The primary end points of this phase II trial were acute toxicities and 2-year late toxicities of esophagus and lungs. The secondary end points included 2-year LRC, DMFS, DFS and OS rates plus the first site of failure. In RTOG 85-01 trial, the 2-year LRC for patients underwent standard-dose RT and chemotherapy was approximately $50 \%$ [1]. Assuming the local control rate for patients enrolled in this study is $65 \%, 85$ patients will be needed with an $80 \%$ power and $5 \%$ of potential early dropout or loss to follow-up. The toxicities and survival of the first 60 patients were summarized in this report. 


\section{Statistical methods}

Toxicities and survivals were measured from the beginning of treatment until the date of events (first site of failure or death) or the last clinic visit. The Statistical Package for Social Sciences (SPSS 21.0, Chicago, IL) was used to calculate the LRC, DMFS, DFS and OS using the Kaplan-Meier method.

\section{ACKNOWLEDGMENTS}

We would like to thank Prof. Albert Koong from Department of Radiation Oncology, Stanford University for his constructive comments. The abstract of this report has been partly presented at the $56^{\text {th }}$ annual meeting of American Society for Radiation Oncology, San Antonio, TX, USA.

\section{FUNDING}

This study was supported by Collaborative and Creative Center, Molecular Diagnosis and Personalized Medicine, Shantou University, Guangdong Province, China, Shantou University Medical College Clinical Research Enhancement Initiative N0201424, and Research and Travel Grants from the Science and Technology Key Project of Shantou City, China, 2012, No. 2012-165-131.

\section{CONFLICTS OF INTEREST}

The authors have declared no competing interests.

\section{REFERENCES}

1. Cooper JS, Guo MD, Herskovic A, Macdonald JS, Martenson JA, Jr., Al-Sarraf M, Byhardt R, Russell AH, Beitler JJ, Spencer S, Asbell SO, Graham MV and Leichman LL. Chemoradiotherapy of locally advanced esophageal cancer: long-term follow-up of a prospective randomized trial (RTOG 85-01). Radiation Therapy Oncology Group. Jama. 1999; 281:1623-1627.

2. Smith TJ, Ryan LM, Douglass HO, Jr., Haller DG, Dayal Y, Kirkwood J, Tormey DC, Schutt AJ, Hinson J and Sischy B. Combined chemoradiotherapy vs. radiotherapy alone for early stage squamous cell carcinoma of the esophagus: a study of the Eastern Cooperative Oncology Group. International journal of radiation oncology, biology, physics. 1998; 42:269-276.

3. Minsky BD, Pajak TF, Ginsberg RJ, Pisansky TM, Martenson J, Komaki R, Okawara G, Rosenthal SA and Kelsen DP. INT 0123 (Radiation Therapy Oncology Group 94-05) phase III trial of combined-modality therapy for esophageal cancer: high-dose versus standard-dose radiation therapy. Journal of clinical oncology. 2002; 20:1167-1174.
4. Lin SH, Wang L, Myles B, Thall PF, Hofstetter WL, Swisher SG, Ajani JA, Cox JD, Komaki R and Liao Z. Propensity score-based comparison of long-term outcomes with 3-dimensional conformal radiotherapy vs intensitymodulated radiotherapy for esophageal cancer. International journal of radiation oncology, biology, physics. 2012; 84:1078-1085

5. Zhang Z, Liao Z, Jin J, Ajani J, Chang JY, Jeter M, Guerrero T, Stevens CW, Swisher S, Ho L, Yao J, Allen P, Cox JD and Komaki R. Dose-response relationship in locoregional control for patients with stage II-III esophageal cancer treated with concurrent chemotherapy and radiotherapy. International journal of radiation oncology, biology, physics. 2005; 61:656-664.

6. Geh JI, Bond SJ, Bentzen SM and Glynne-Jones R. Systematic overview of preoperative (neoadjuvant) chemoradiotherapy trials in oesophageal cancer: evidence of a radiation and chemotherapy dose response. Radiotherapy and oncology. 2006; 78:236-244.

7. Withers HR, Taylor JM and Maciejewski B. The hazard of accelerated tumor clonogen repopulation during radiotherapy. Acta Oncol. 1988; 27:131-146.

8. Taylor JM, Withers HR and Mendenhall WM. Dose-time considerations of head and neck squamous cell carcinomas treated with irradiation. Radiotherapy and oncology. 1990; 17:95-102.

9. Nishimura Y, Ono K, Tsutsui K, Oya N, Okajima K, Hiraoka $M$ and Abe $M$. Esophageal cancer treated with radiotherapy: impact of total treatment time and fractionation. International journal of radiation oncology, biology, physics. 1994; 30:1099-1105.

10. Kajanti M, Kaleta R, Kankaanranta L, Muhonen T and Holsti L. Effect of overall treatment time on local control in radical radiotherapy for squamous cell carcinoma of esophagus. International journal of radiation oncology, biology, physics. 1995; 32:1017-1023.

11. Zhang WZ, Chen JZ, Li DR, Chen ZJ, Guo H, Zhuang TT, Li DS, Zhou MZ and Chen CZ. Simultaneous modulated accelerated radiation therapy for esophageal cancer: A feasibility study. World journal of gastroenterology : WJG. 2014; 20:13973-13980.

12. Prescribing, Recording, and Reporting Photon-Beam Intensity-Modulated Radiation Therapy (IMRT): Contents. Journal of the ICRU. 2010; 10:NP.

13. Warren S, Partridge M, Carrington R, Hurt C, Crosby $\mathrm{T}$ and Hawkins MA. Radiobiological determination of dose escalation and normal tissue toxicity in definitive chemoradiation therapy for esophageal cancer. International journal of radiation oncology, biology, physics. 2014; 90:423-429.

14. Welsh J, Palmer MB, Ajani JA, Liao Z, Swisher SG, Hofstetter WL, Allen PK, Settle SH, Gomez D, Likhacheva A, Cox JD and Komaki R. Esophageal cancer dose escalation using a simultaneous integrated boost technique. 
International journal of radiation oncology, biology, physics. 2012; 82:468-474.

15. Conroy T, Galais MP, Raoul JL, Bouche O, GourgouBourgade S, Douillard JY, Etienne PL, Boige V, MartelLafay I, Michel P, Llacer-Moscardo C, Francois E, Crehange $G$, et al. Definitive chemoradiotherapy with FOLFOX versus fluorouracil and cisplatin in patients with oesophageal cancer (PRODIGE5/ACCORD17): final results of a randomised, phase $2 / 3$ trial. The Lancet Oncology. 2014; 15:305-314

16. Gaspar LE, Winter K, Kocha WI, Coia LR, Herskovic A and Graham M. A phase I/II study of external beam radiation, brachytherapy, and concurrent chemotherapy for patients with localized carcinoma of the esophagus (Radiation Therapy Oncology Group Study 9207): final report. Cancer. 2000; 88:988-995.

17. Wani S, Das A, Rastogi A, Drahos J, Ricker W, Parsons R, Bansal A, Yen R, Hosford L, Jankowski M, Sharma P and Cook MB. Endoscopic ultrasonography in esophageal cancer leads to improved survival rates: results from a population-based study. Cancer. 2015; 121:194-201.

18. Thomas E, Crellin A, Harris K, Swift S and Montefiore DS. The role of endoscopic ultrasound (EUS) in planning radiotherapy target volumes for oesophageal cancer. Radiotherapy and oncology. 2004; 73:149-151.
19. Yap JC, Malhotra HK and Yang GY. Intensity modulated radiation therapy in the treatment of esophageal cancer. Thorac Cancer. 2010; 1:62-69.

20. Cao C, Luo J, Gao L, Xu G, Yi J, Huang X, Wang K, Zhang S, Qu Y, Li S, Xiao J and Zhang Z. Definitive intensity-modulated radiotherapy compared with definitive conventional radiotherapy in cervical oesophageal squamous cell carcinoma. La Radiologia medica. 2015; 120:603-610.

21. Freilich J, Hoffe SE, Almhanna K, Dinwoodie W, Yue B, Fulp W, Meredith KL and Shridhar R. Comparative outcomes for three-dimensional conformal versus intensitymodulated radiation therapy for esophageal cancer. Diseases of the esophagus. 2015; 28:352-357.

22. Yu W, Cai XW, Liu Q, Zhu ZF, Feng W, Zhang Q, Zhang YJ, Yao ZF and Fu XL. Safety of dose escalation by simultaneous integrated boosting radiation dose within the primary tumor guided by (18)FDG-PET/CT for esophageal cancer. Radiotherapy and oncology. 2015; 114:195-200.

23. Greene FL, American Joint Committee on Cancer and American Cancer Society. (2002). AJCC cancer staging manual. (New York: Springer-Verlag).

24. National Cancer Institute (U.S.). (2009). Common terminology criteria for adverse events (CTCAE). (Bethesda, Md.: U.S. Dept. of Health and Human Services, National Institutes of Health, National Cancer Institute). 